\title{
Decompressive craniectomy for internal carotid artery and middle carotid artery infarctions: a long-term comparative outcome study
}

\author{
Jia Xu Lim, MRCS, ${ }^{1}$ Srujana Venkata Vedicherla, MRCS, ${ }^{1}$ Shu Kiat Sukit Chan, MRCS, ${ }^{1}$ \\ Nishal Kishinchand Primalani, MRCS, ${ }^{1}$ Audrey J. L. Tan, MRCS, ${ }^{1}$ Seyed Ehsan Saffari, PhD, ${ }^{2}$ and \\ Lester Lee, FRCS(SN) ${ }^{1,3}$
}

${ }^{1}$ Department of Neurosurgery, National Neuroscience Institute; and ${ }^{2}$ Centre for Quantitative Medicine, ${ }^{3}$ Duke-NUS Medical School, Singapore

OBJECTIVE Malignant internal carotid artery (ICA) infarction is an entirely different disease entity when compared with middle cerebral artery (MCA) infarction. Because of an increased area of infarction, it is assumed to have a poorer prognosis; however, this has never been adequately investigated. Decompressive craniectomy (DC) for malignant MCA infarction has been shown to improve mortality rates in several randomized controlled trials. Conversely, aggressive surgical decompression for ICA infarction has not been recommended. The authors sought to compare the functional outcomes and survival between patients with ICA infarctions and those with MCA infarctions after DC in the largest series to date to investigate this assumption.

METHODS A multicenter retrospective review of 154 consecutive DCs for large territory cerebral infarctions performed from 2005 to 2020 were analyzed. Patients were divided into ICA and MCA groups depending on the territory of infarction. Variables, including age, sex, medical comorbidities, laterality of the infarction, preoperative neurological status, primary stroke treatment, and the time from stroke onset to DC, were recorded. Univariable and multivariable analyses were performed for the clinical exposures for functional outcomes (modified Rankin Scale [mRS] score) on discharge and at the 1-and 6-month follow-ups, and for mortality, both inpatient and at the 1-year follow-up. A favorable mRS score was defined as $0-2$.

RESULTS There were 67 patients (43.5\%) and 87 patients (56.5\%) in the ICA and MCA groups, respectively. Univariable analysis showed that the ICA group had a comparably favorable mRS (OR $0.15[95 \% \mathrm{Cl} 0.18-1.21], \mathrm{p}=0.077)$. Inpatient mortality (OR 1.79 [95\% Cl 0.79-4.03], $p=0.16$ ) and 1-year mortality (OR 2.07 [95\% Cl 0.98-4.37], $p=$ $0.054)$ were comparable between the groups. After adjustment, a favorable mRS score at 6 months $(\mathrm{OR} 0.17[95 \% \mathrm{Cl}$ 0.018-1.59], $p=0.12$ ), inpatient mortality (OR 1.02 [95\% Cl 0.29-3.57], $p=0.97)$, and 1-year mortality (OR 0.94 [95\% Cl $0.41-2.69], p=0.88$ ) were similar in both groups. The overall survival, plotted using the Cox proportional hazard regression, did not show a significant difference between the ICA and MCA groups (HR 0.581).

CONCLUSIONS Unlike previous smaller studies, this study found that patients with malignant ICA infarction had a functional outcome and survival that was similar to those with MCA infarction after DC. Therefore, DC can be offered for malignant ICA infarction for life-saving purposes with limited functional recovery.

https://thejns.org/doi/abs/10.3171/2021.4.FOCUS21123

KEYWORDS decompressive craniectomy; surgical decompression; stroke; functional outcome; survival

$\mathrm{T}$ HE treatment of stroke has been revolutionized in recent decades by significant progress in thrombectomy, ${ }^{1}$ neuroimaging, ${ }^{2}$ and improved access to medical therapy. ${ }^{3}$ However, certain held beliefs in stroke management, such as the futility of decompressive crani- ectomy (DC) for internal carotid artery (ICA) cerebral infarctions, have never been adequately investigated.

ICA infarction is relatively less common than middle cerebral artery (MCA) infarction, accounting for $19.5 \%$ and $65 \%$ of large-vessel occlusive strokes, respectively. ${ }^{4}$

ABBREVIATIONS ACA = anterior cerebral artery; $\mathrm{DC}=$ decompressive craniectomy; $\mathrm{CCI}=$ Charlson Comorbidity Index; $\mathrm{GCS}=\mathrm{Glasgow}$ Coma Scale; ICA = internal carotid artery; ICP = intracranial pressure; $M C A=$ middle cerebral artery; $m R S=$ modified Rankin Scale; NICU = neurosurgical intensive care unit; $P C A=$ posterior cerebral artery.

SUBMITTED February 28, 2021. ACCEPTED April 6, 2021

INCLUDE WHEN CITING DOI: 10.3171/2021.4.FOCUS21123. 
The pathophysiology of an ICA infarction is similar to that of an MCA infarction, although the affected territory is larger. Occlusion of the vessel results in the interruption of blood flow in the areas of the brain perfused by it. The resultant cell death causes malignant cerebral edema in the acute to subacute period, which leads to raised intracranial pressure. This in turn results in uncal, transtentorial herniation, and ultimately, tonsillar herniation, leading to death. ${ }^{5}$ In ICA infarction, with a larger volume of brain infarcted (i.e., MCA with anterior cerebral artery [ACA] and/or posterior cerebral artery [PCA] territory), more cerebral edema is produced, and intracranial pressure (ICP) is increased compared with that of MCA infarction. ${ }^{6}$ The current treatment of ICA occlusion is aimed at preventing the establishment of an infarct by recanalization of the vessel and restoration of cerebral perfusion. The use of intravenous thrombolytics have not proven to be greatly beneficial, ${ }^{7}$ and the mainstay of current treatment is endovascular thrombectomy with or without stenting., ${ }^{4,-9}$

Malignant cerebral edema can lead to a mortality rate of $80 \%$ when treated medically. ${ }^{10,11}$ DC has been established as a treatment for malignant cerebral edema for strokes, but its use has mainly been focused on MCA infarction. ${ }^{10,12-19}$ Various randomized controlled trials have shown a significant improvement in the survival of patients who underwent DC for malignant MCA infarction, ${ }^{20-22}$ from $29 \%$ to $78 \%$. However, an improvement in functional outcomes was not demonstrated. ${ }^{23}$ Previous studies have also found that significant predictors of mortality and poor functional outcome in such patients included older age, previous history of stroke, diabetes mellitus, preoperative low Glasgow Coma Scale (GCS) score, the presence of anisocoria, a midline shift $>10 \mathrm{~mm}$, and an increased time from the onset of stroke to DC. ${ }^{12,24-28}$

The aim of this study was to determine if DC is as effective in reducing mortality and morbidity in patients with malignant ICA infarction compared with those with malignant MCA infarction and to review the long-term outcomes in both groups of patients.

\section{Methods \\ Study Design}

This multicenter retrospective study was conducted in Tan Tock Seng Hospital and Singapore General Hospital, two of the largest neurosurgical units in Singapore, a multicultural Asian city. From January 2005 to March 2020, all consecutive patients who were admitted for large territory infarction in the MCA or ICA territory and subsequently underwent therapeutic DC for malignant cerebral edema were recruited. Institutional ethics review board was obtained.

\section{Inclusion and Exclusion Criteria}

Patients aged 18 to 95 years who underwent therapeutic DC for either an MCA or ICA territory infarction at our two main hospitals were included. An MCA infarction was defined as cerebral infarction occurring within the MCA territory, while an ICA infarction was defined as cerebral infarction occurring within the ACA or PCA territory in addition to the MCA territory. These patients were select- ed based on the institutional protocol determined by the neurosurgical and neurology departments. The inclusion criteria included having an acute large territory infarction involving more than $50 \%$ of MCA territory on imaging; a premorbid modified Rankin Scale ( $\mathrm{mRS}$ ) score of $<3$; and clinical deterioration according to the GCS score, pupillary reaction, or progression of focal neurological deficit. Patients who were comatose with a GCS score $<6$, those with fixed and dilated bilateral pupils, those with serious medical comorbidities that precluded surgery, and those with a likely life expectancy $<3$ years were excluded. Our study also excluded patients who underwent prophylactic DC and patients with a large territory infarction due to surgical complications or trauma.

\section{Surgical Procedure and Periprocedural Care}

On admission, patients meeting the American Heart Association/American Stroke Association stroke guidelines ${ }^{17}$ underwent thrombolytic intravenous therapy with recombinant tissue plasminogen activator (rtPA) and/or thrombectomy. Patients were subsequently admitted to a dedicated stroke unit or high-dependency ward. On satisfying the abovementioned inclusion and exclusion criteria, therapeutic DC was offered.

Decompressive hemicraniectomy was performed as follows: a large reverse-question-mark skin incision was performed ipsilateral to the side of the stroke. A large bone flap with a diameter of at least $12 \mathrm{~cm}$ was removed, with additional subtemporal decompression performed. The dura mater was opened in a linear, curvilinear, or stellate fashion. An ICP monitor was inserted to allow ICP-guided management postoperatively. Closure was performed with a dural substitute that was either secured in a loose fashion or laid over the original dura. Epidural drains were inserted, and soft tissue was closed in layers.

All patients remained intubated and sedated postoperatively and were managed in a dedicated neurosurgical intensive care unit (NICU). Medical management was then instituted as required to control ICP postoperatively (targeting $<20 \mathrm{~mm} \mathrm{Hg}$ ) with sedation, osmotic diuretics, and barbiturate coma.

\section{Data Collection}

Records and imaging files were reviewed for the current analysis, and clinical, radiological, and monitoring data were extracted. The recruited patients were classified into two groups based on the territory of the ischemic infarction: an MCA infarction group (limited to the MCA territory) and an ICA infarction group (extended to involve the MCA and ipsilateral ACA and/or PCA territory). Patient characteristics, including age, sex, past medical history formulated using the Charlson Comorbidity Index (CCI), laterality of the infarction, and the time elapsed from stroke onset to decompressive surgery, were recorded. The clinical status of each patient was assessed both pre- and postoperatively by using the GCS and by observing the presence of pupillary dilation. The primary treatment of the stroke with either thrombolysis or thrombectomy was also documented.

Outcome measures, including the length of stay in the 
NICU (days) and the overall hospital stay (from admission to discharge or death, in days), postoperative morbidity and mortality during the follow-up duration, and function, were documented. Dates of death (including both inpatient and outside the hospital) were obtained from the Singapore National Registry of Diseases Office. De-identification of our patient data set was also performed by the Singapore National Registry of Diseases Office. Functional outcome was assessed using the mRS score at discharge and at 1 month and 6 months after the procedure. In our study, a CCI score $\leq 2$, a GCS score $>8$, and an mRS score of $0-2$ were defined as favorable. The mean follow-up duration was formulated based on attendance at follow-up visits.

\section{Statistical Analysis}

Variable distributions are described using frequency (\%) and mean \pm standard deviation or median (IQR) for categorical and continuous variables, respectively. The two patient groups were compared using the chi-square test (or the Fisher's exact test, where appropriate) and the two-sample t-test (or the Mann-Whitney U-test, depending on the normality assumption) for categorical and continuous variables, respectively, for statistical significance.

Multivariable logistic regression models with Firth's penalized likelihood were performed to investigate outcomes, including the 6-month $\mathrm{mRS}$ score, inpatient mortality, and 1-year mortality, after adjusting for potential confounders identified by either clinical literature or univariable analysis. A Cox proportional hazard survival curve was plotted, and the hazard ratio was used to assess the difference between the survival curve. Statistical significance was set at $p<0.05$. Data analyses were performed using IBM SPSS Statistics version 20.0 (IBM Corp.).

\section{Results \\ Patient Characteristics}

A total of 154 therapeutic DCs were performed during the study period; 67 were performed in patients with an ICA infarct, and 87 were performed in patients with an MCA infarct. Both groups were comparable in terms of the mean age (ICA, $56.7 \pm 11.3$ years; MCA, $58.5 \pm 12.6$ years), sex (ICA, 65.7\% male; MCA, 69.0\% male), medical comorbidities (median CCI score 2 for both groups), preoperative neurological status (the median GCS score 9 for both groups), the presence of anisocoria (ICA, 19.4\%; MCA, 12.6\%), primary stroke treatment such as thrombolysis (ICA, 34.3\%; MCA, 35.6\%) and thrombectomy (ICA, 4.5\%; MCA, 4.6\%), and the mean time from onset of the stroke to decompressive surgery (ICA, $42.1 \pm 28.7$ hours; MCA, $39.6 \pm 26.6$ hours). Of note, the proportion of left-sided surgeries between the two groups approached significance (ICA, 31.3\%; MCA, 46.0\%; $\mathrm{p}=0.066$ ). These characteristics are summarized in Table 1 .

\section{Inpatient Postoperative Outcomes}

With regard to postoperative outcomes, the median postoperative GCS score (ICA, 9 and MCA, 11; $p=0.045$ ) and percentage of patients with postoperative anisocoria (ICA, $14.9 \%$ and MCA, $4.6 \%$; $\mathrm{p}=0.045$ ) were signifi-
TABLE 1. Characteristics of the ICA and MCA patient groups

\begin{tabular}{lccc}
\hline & $\begin{array}{c}\text { ICA Infarct } \\
\text { Group }(\mathrm{n}=67)\end{array}$ & $\begin{array}{c}\text { MCA Group } \\
(\mathrm{n}=87)\end{array}$ & $\begin{array}{c}\mathrm{p} \\
\text { Value }\end{array}$ \\
\hline Age, yrs & & & 0.35 \\
\hline Range & $29-77$ & $24-92$ & \\
\hline Mean \pm SD & $56.7 \pm 11.3$ & $58.5 \pm 12.6$ & 0.67 \\
\hline Sex, $\mathrm{n}(\%)$ & & & \\
\hline $\mathrm{M}$ & $44(65.7)$ & $60(69.0)$ & \\
\hline $\mathrm{F}$ & $23(34.3)$ & $27(31.0)$ & \\
\hline Median CCI score (IQR) & $2(1-3)$ & $2(1-3)$ & 0.81 \\
\hline Laterality, $\mathrm{n}(\%)$ & & & 0.066 \\
\hline Lt & $21(31.3)$ & $40(46.0)$ & \\
\hline Rt & $46(68.7)$ & $47(54.0)$ & \\
\hline $\begin{array}{l}\text { Median preop GCS score } \\
\text { (IQR) }\end{array}$ & $9(8-10.75)$ & $9(8-11)$ & 0.42 \\
\hline Preop anisocoria, $\mathrm{n}(\%)$ & $13(19.4)$ & $11(12.6)$ & 0.25 \\
\hline Thrombolysis, $\mathrm{n}(\%)$ & $23(34.3)$ & $31(35.6)$ & 0.87 \\
\hline Thrombectomy, $\mathrm{n}(\%)$ & $3(4.5)$ & $4(4.6)$ & $>0.99$ \\
\hline Time to decompression, hrs & & & 0.58 \\
\hline $\begin{array}{l}\text { Range } \\
\text { Mean } \pm \text { SD }\end{array}$ & $8-408$ & $6-264$ & \\
\hline
\end{tabular}

${ }^{*}$ Chi-square test (or Fisher exact test, where appropriate) and two sample t-test (or Mann Whitney U-test, depending on normality assumption) for categorical and continuous variables, respectively.

cantly different. The median overall length of stay in the NICU (ICA, 8 days; MCA, 6 days) and the median overall hospital stay (ICA, 40 days; MCA, 30 days), the need for tracheostomy (ICA, 20.9\%; MCA, 18.4\%), and the rate of complications (ICA, 14.9\%; MCA, 6.9\%) were comparable. The proportion of inpatient deaths (ICA, 23.9\%; MCA, 14.9\%) and a favorable mRS score at the time of discharge (ICA, $1.5 \%$; MCA, $1.1 \%$ ) were also comparable. However, patients with an MCA infarct were more likely to go home or to a rehabilitation facility on discharge (MCA, 63.2; ICA, 43.3\%; $\mathrm{p}=0.017$ ). These outcomes are detailed in Table 2.

\section{Long-Term Outcomes}

Both groups of patients underwent follow-up for an adequate duration; the median duration for the ICA group was 24 months and for the MCA group, 36 months. The difference in mortality at the 1-year interval trended toward significance (ICA, 35.5\%; MCA, 21\%; $\mathrm{p}=0.054$ ). When considering functional outcomes, favorable mRS scores were similar between the groups at the 1-month (ICA, 1.6\% and MCA, 2.4\%; p > 0.99) and 6-month (ICA, $1.8 \%$ and MCA, 10.8\%; $\mathrm{p}=0.077)$ marks. The long-term outcomes highlighted in Table 3 and Fig. 1 demonstrate the number of patients included in our analysis at each critical timepoint.

\section{Functional Outcome and Mortality}

When considering inpatient mortality, having good preoperative (HR 0.36 [95\% CI 0.16-0.84], $\mathrm{p}=0.015$ ) and 
TABLE 2. Inpatient outcomes of the ICA and MCA groups

\begin{tabular}{lccc}
\hline & $\begin{array}{c}\text { ICA Infarct } \\
\text { Group } \\
(\mathrm{n}=67)\end{array}$ & $\begin{array}{c}\text { MCA Infarct } \\
\text { Group } \\
(\mathrm{n}=87)\end{array}$ & $\begin{array}{c}\mathrm{p} \\
\text { Value }\end{array}$ \\
\hline Median postop GCS score & $9(6-12)$ & $11(9-12)$ & $\mathbf{0 . 0 4 5}$ \\
\hline Postop anisocoria & $10(14.9)$ & $4(4.6)$ & $\mathbf{0 . 0 4 5}$ \\
\hline Median NICU LOS, days & $8(5-11)$ & $6(4-10)$ & 0.081 \\
\hline Median overall LOS, days & $40(21-60)$ & $30(20-49)$ & 0.18 \\
\hline Tracheostomy & $14(20.9)$ & $16(18.4)$ & 0.70 \\
\hline Complications & $10(14.9)$ & $6(6.9)$ & 0.11 \\
\hline Inpatient mortality & $16(23.9)$ & $13(14.9)$ & 0.16 \\
\hline Favorable discharge mRS score & $1(1.5)$ & $1(1.1)$ & $>0.99$ \\
\hline Discharge location & & & 0.017 \\
\hline \multicolumn{1}{c}{ Home/rehab facility } & $29(43.3)$ & $55(63.2)$ & \\
\hline Nursing facility & $18(26.5)$ & $10(11.4)$ & \\
\hline Repatriation & $4(6.0)$ & $8(9.2)$ \\
\hline
\end{tabular}

LOS = length of stay.

Values represent the number of patients (\%) unless stated otherwise. Median values are presented as the median (IQR). Boldface type indicates statistical significance.

postoperative (HR 0.047 [0.016-0.14], p < 0.0001) GCS score was protective against inpatient mortality, while having preoperative (HR 8.42 [95\% CI 3.29-22.34], $\mathrm{p}<$ 0.0001 ) and postoperative (HR 10.8 [95\% CI 3.28-35.5], $\mathrm{p}<0.0001$ ) anisocoria and a previous thrombectomy (HR 12.8 [95\% CI 2.35-69.9], $\mathrm{p}=0.003$ ) were associated with inpatient death. After adjusting for confounders, the postoperative GCS score (HR 0.035 [95\% CI 0.008-0.15], p < 0.0001 ), preoperative anisocoria (HR 7.12 [95\% CI 1.6031.7], $\mathrm{p}=0.010$ ), and having had a thrombectomy (HR 19.1 [95\% CI 1.73-210], $\mathrm{p}=0.016$ ) remained statistically significant. ICA infarction, before and after adjustment, was not associated with inpatient mortality.

Factors associated with a favorable 6-month mRS score included age $<60$ years (OR 7.49 [95\% CI 0.91-61.7], p $=0.038)$ and a favorable CCI score (OR $7.02[95 \%$ CI $0.85-57.8], p=0.043$ ). Having an ICA rather than an MCA infarction was not associated with an unfavorable 6-month mRS score before (OR 0.15 [95\% CI 0.18-1.21, $\mathrm{p}=0.077$ ] and after (OR 0.17 [95\% CI 0.018-1.59], $\mathrm{p}=$ 0.12 ) adjustment. Having a left-sided infarct also had no association with the 6-month $\mathrm{mRS}$ score both without (OR 0.21 [95\% CI 0.025-1.71], $\mathrm{p}=0.16$ ) and with (OR
0.12 [95\% CI 0.013-1.12], $\mathrm{p}=0.063$ ) adjustment. Figure 2 gives a graphical representation of the mRS score at 6 months when considering both the extent and laterality of the infarction (a favorable mRS score in the right ICA infarction, 1 [2.4\%] and right MCA infarction, 7 [16.7\%]; a favorable $\mathrm{mRS}$ score in the left ICA infarction, 0 [0\%] and left MCA infarction, 1 [3.1\%]).

Variables associated with mortality at the 1-year followup included having a low CCI score (HR 0.42 [95\% CI $0.20-0.89], p=0.021$ ), good preoperative (HR 0.31 [95\% CI 0.14-0.68], $\mathrm{p}=0.002$ ) and postoperative (HR 0.051 [95\% CI 0.020-0.13], $\mathrm{p}<0.0001$ ) GCS score, preoperative (HR 5.91 [95\% CI 2.30-15.2], $\mathrm{p}<0.0001$ ) and postoperative (HR 11.6 [95\% CI 3.00-45.0], p < 0.0001) anisocoria, and a previous thrombectomy (HR 7.50 [95\% CI 1.39 40.4], $\mathrm{p}=0.016)$. After adjustment, having a favorable postoperative GCS score (HR 0.041 [95\% CI 0.012-0.15], $\mathrm{p}<0.0001$ ) and a previous tracheostomy (HR $0.26[95 \%$ CI 0.071-0.52], $\mathrm{p}=0.0023$ ) remained significant. Having an ICA infarction was not associated with 1-year mortality before or after multivariable analysis.

\section{Overall Survival}

When all-cause mortality is considered, the Cox proportional hazard regression does not demonstrate any statistical difference between the ICA and MCA groups (HR 0.581 ) after adjusting for age, side of infarction, time from stroke onset to DC, CCI score, preoperative and postoperative GCS score, and anisocoria (Fig. 3).

\section{Discussion}

There is a paucity of studies in the literature regarding surgical decompression of large territory ICA infarction. Studies on ICA infarction were generally more conservative when recommending surgery compared with MCA infarction, due to findings of higher mortality and poorer functional outcome in ICA infarction. ${ }^{12,15,26-28}$ Nevertheless, these were smaller retrospective studies that may not have captured an adequate spectrum of this disease population. Our study has demonstrated that the long-term functional and survival outcomes of patients who underwent therapeutic DC for ICA infarction were comparable to those of patients who underwent DC for MCA infarction. To the best of our knowledge, this is the largest study of ICA infarction with regard to outcomes after decompressive surgery.

The patients in our study who underwent ICA decompression were selected carefully based on good prognos-

TABLE 3. Long-term outcomes of the ICA and MCA patient groups

\begin{tabular}{|c|c|c|c|c|c|c|}
\hline & $\begin{array}{l}\text { ICA Infarct } \\
(n=67)\end{array}$ & $\begin{array}{c}\text { MCA Infarct } \\
(\mathrm{n}=87)\end{array}$ & $\begin{array}{c}\text { OR } \\
(95 \% \mathrm{Cl})\end{array}$ & $\begin{array}{c}p \\
\text { Value }\end{array}$ & $\begin{array}{l}\text { Adjusted OR } \\
(95 \% \mathrm{Cl})\end{array}$ & $\begin{array}{c}\text { Adjusted } \\
\text { p Value }\end{array}$ \\
\hline Favorable mRS score at $1 \mathrm{mo}$ & $1 / 62(1.6)^{*}$ & $2(2.4)^{*}$ & $0.65(0.058-7.40)$ & $>0.99$ & $0.95(0.060-14.0)$ & 0.95 \\
\hline Favorable mRS score at 6 mos & $1 / 57(1.8)^{*}$ & $8(10.8)^{*}$ & $0.15(0.18-1.21)$ & 0.077 & $7.15(0.74-69.4)$ & 0.090 \\
\hline 1-yr mortality & $22(35.5)$ & $17(21.0)$ & $2.07(0.98-4.37)$ & 0.054 & $0.95(0.32-2.81)$ & 0.92 \\
\hline Median follow-up duration (IQR), mos & $24(18-39.75)$ & $36(21.5-48)$ & & 0.20 & & \\
\hline
\end{tabular}

* Presented as the number of patients/total number of patients available at the stated time point. 


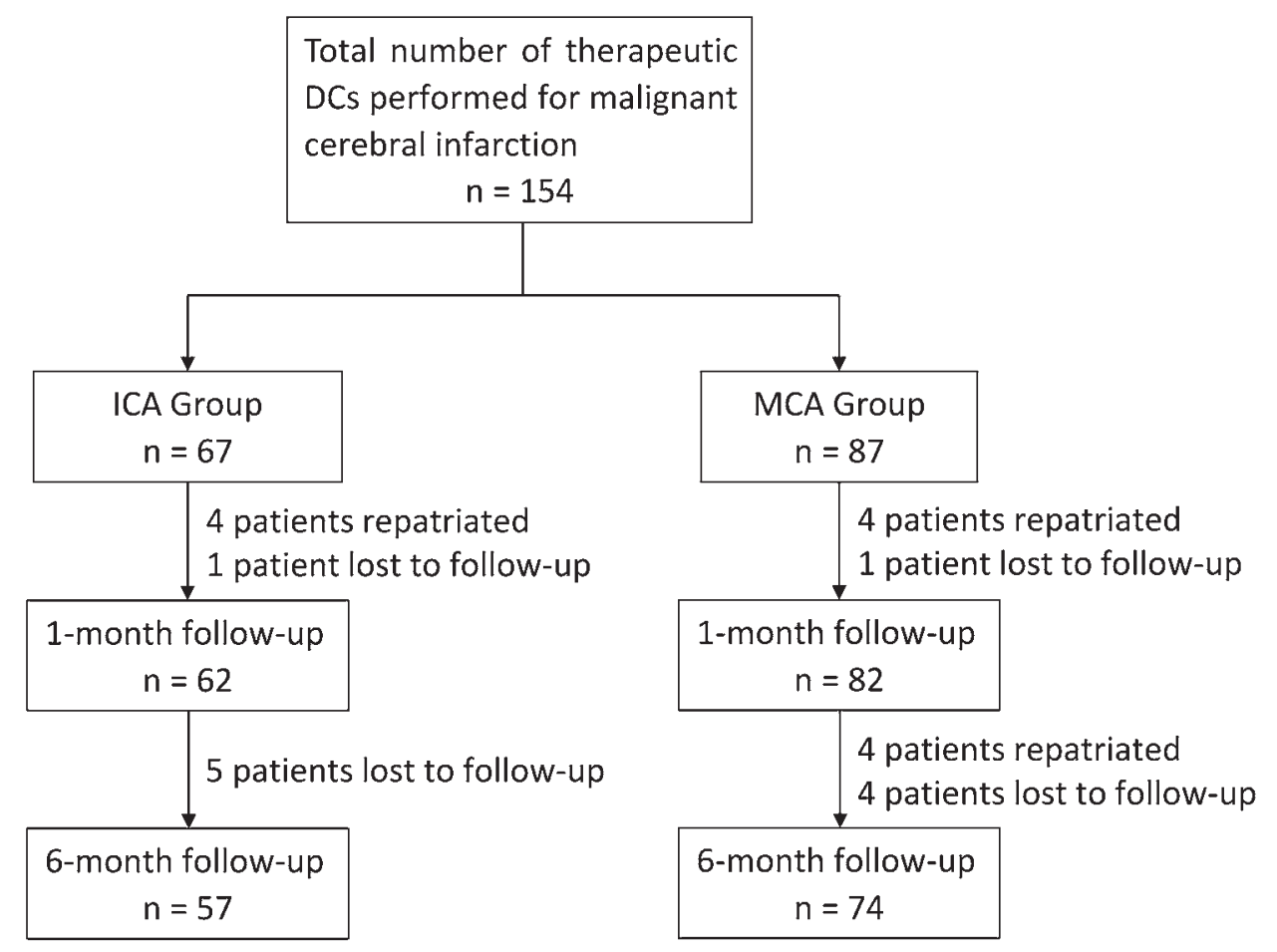

FIG. 1. Flowchart of the patients at various time points, and the outcome evaluation at the 1-month and 6-month follow-ups after DC.

tic factors in DC for MCA infarction as described in the literature. Younger age, lower CCI score, an acceptable preoperative GCS score, and a low proportion of patients with anisocoria are characteristics that reflect the relatively good premorbid and neurological status of patients selected for surgery. Fewer left-sided surgeries in the ICA group reflects the perception that large territory dominant hemispheric infarcts tend to have a poorer neurological and hence functional prognosis. ${ }^{29}$

The ICA group had a worse median postoperative GCS score and persistent anisocoria after decompression, which demonstrates more severe swelling and higher ICP exerted by the infarcted ICA territory compared with the MCA territory. Despite this, the length of stay in the NICU and inpatient morbidity and mortality were comparable in both patient groups. In addition, after adjustment, a favorable mRS score on discharge and at the 1- and 6-month follow-up, as well as 1-year mortality, were similar among both groups; this was despite a significantly larger proportion of ICA infarction patients being discharged to nursing homes, rather than to home or a rehabilitation facility. The overall survival, when considering all-cause mortality, was also comparable between the two groups.

Our study challenges the conventional thinking that ICA infarctions inherently herald a worse functional prognosis and survival despite DC. This notion is associated with the condition having a much larger territory of affected brain tissue, leading to increased cerebral edema. ${ }^{6}$ However, damage to the functional anatomy within the ACA territory may not be reflected in the overall functional status of the patient. Higher cortical function in the ACA territory such as planning, motivation, mood, and emotions $\mathrm{s}^{30,31}$ may not directly factor into the ability to perform basic self-care and ambulation. DC, in conjunction with modern ICP-lowering methods, is sufficient to compensate for the increased swelling from the additional region involved in an ICA infarction. Therefore, should the surgical aim of the therapeutic DC be solely for life-saving purposes, having an ICA infarction should not be a deterrent for aggressive surgical intervention.

When comparing the ICA group patients in our study with those presented in the literature, ${ }^{12,15,26-28}$ the former had less mortality and better functional status over a longer follow-up interval. Patients with ICA infarctions who underwent DC in our study had an inpatient mortality of $23.5 \%$ and a 1-year mortality of $34.9 \%$ compared with $37.5 \%$ to $70 \% 12,15,26,27$ in other studies. This is likely related to better patient selection with a younger population, fewer medical comorbidities, and a better preoperative neurological status. In addition, many of these studies reported on patients who underwent surgical decompression of their ICA infarction prior to 2005. Although the surgical technique has not changed, the anesthesia techniques 32,33 and perioperative care ${ }^{34,35}$ have advanced, and the philosophy of patient selection has shifted, further improving outcomes. There may also be the effect of "self-fulfilling prophecy" seen in other neurosurgical conditions such as intracerebral hematomas, where prognostic pessimism is associated with early withdrawal of care, ${ }^{36,37}$ possibly leading to the "predicted" poor outcomes. ${ }^{38}$

On the other hand, our study demonstrated that leftsided infarction showed a trend toward significance, after adjustment, when correlated with an unfavorable 6-month mRS score. More than $90 \%$ of people have a left-hemi- 


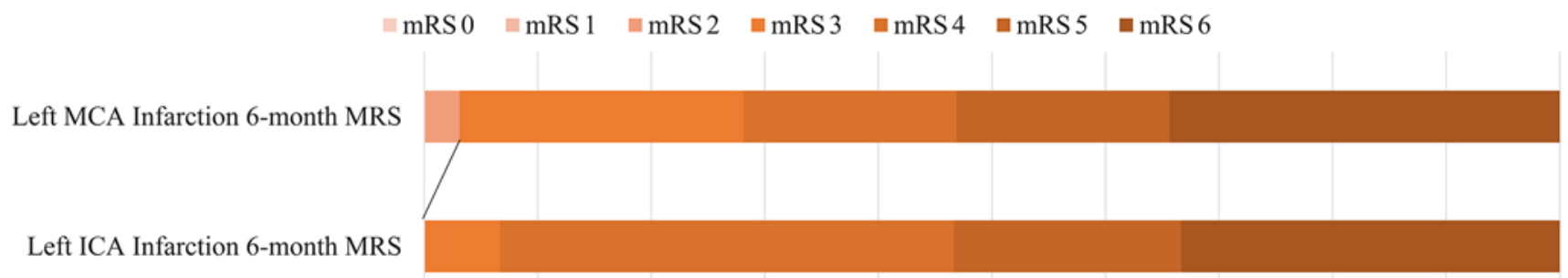

Right MCA Infarction 6-month MRS

Anfarction 6-month MRS

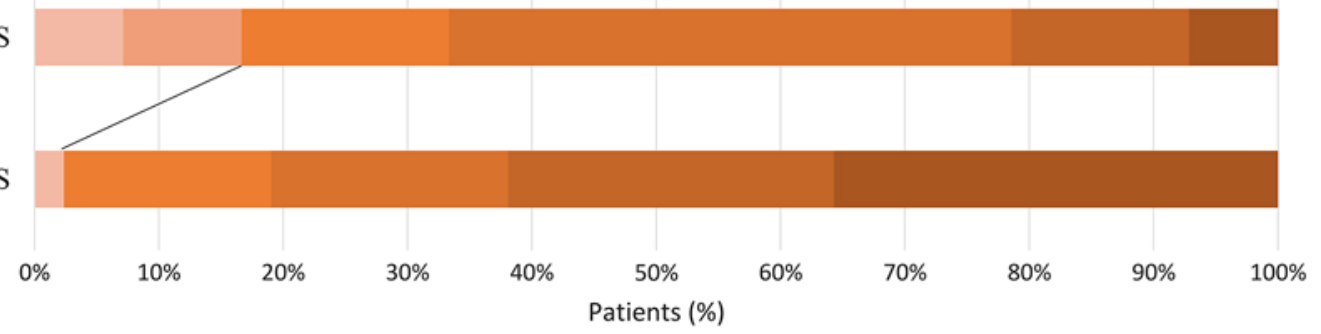

FIG. 2. Comparison of 6 -month mRS scores between left- and right-sided infarctions. The solid black line denotes the mRS score threshold between favorable (0-2) and unfavorable (3-6) outcomes.

spheric language dominance. ${ }^{39}$ The mRS has a global nature that allows the indirect assessment of nonphysical attributes that contribute to well-being, such as language. ${ }^{40}$ Hence, we advise that the counseling process for surgery should be different and nuanced when a dominant hemispheric infarction is concerned.

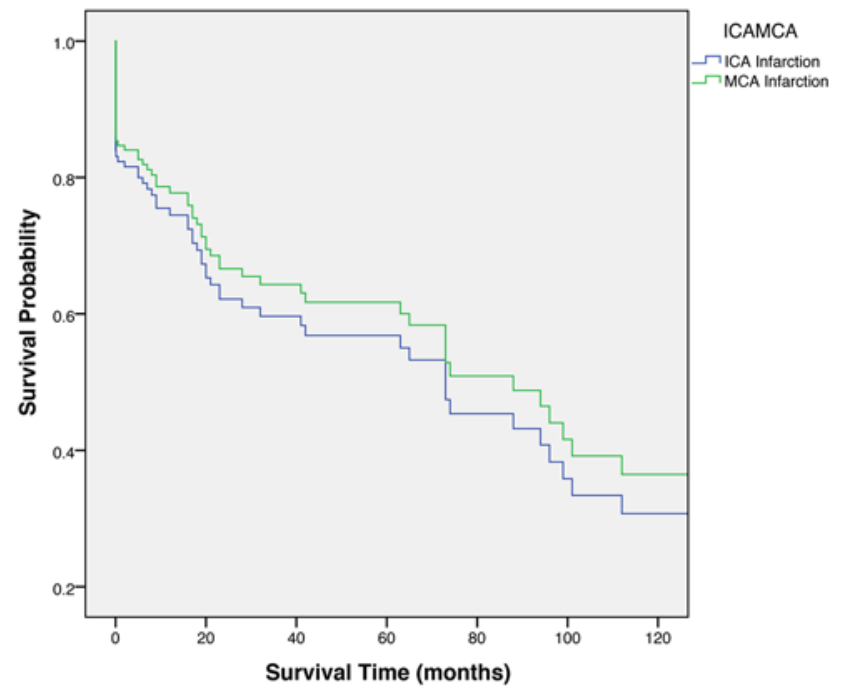

FIG. 3. Adjusted survival curve using the Cox proportional hazard regression demonstrating no difference in survival between the ICA infarct (blue) and MCA infarct (green) patient groups. Adjusted HR = 0.581 . Factors included in the multivariable analysis were age, side of infarction, time to decompression, $\mathrm{CCl}$ score, pre- and postoperative GCS scores, and presence of anisocoria.
Previous studies have suggested that DC for malignant infarctions "converts mortality into severe morbidity" and as such has been criticized by some to be an exercise in futility, ${ }^{41}$ and others even consider severe disability to be a fate worse than death..$^{42}$ However, when surveys were conducted, the results were encouraging; approximately $80 \%$ of patients and/or caregivers when interviewed were satisfied with the surgical outcomes and would give consent again for the procedure. In addition, there was no reported difference in the quality of life in patients with dominant versus nondominant infarcts, even when they experienced dysphasia. ${ }^{43,44}$ In our opinion, the definition of a good quality of life is extremely subjective and is influenced by culture, socioeconomic status, and individual life experience. It is the challenge of the neurosurgeon and neurologist to identify, in a timely manner, where the patient and their family set their threshold for a "favorable mRS score" and to deliver care that conforms to the patient's ideas, concerns, and expectations.

Cultural beliefs in the context of our study have had implications on decision-making with the performance prophylactic versus therapeutic decompressive surgery. ${ }^{45-47}$ Within the Asian population, brain surgery is viewed as a major event that should be avoided as much as possible. There is also a strong culture of family, with responsibility felt toward close relatives, in addition to a belief in the sanctity of life above and beyond having a good quality of life. Hence, there is likely to be a larger proportion of therapeutic than prophylactic surgeries and a likelihood for patients to be more accepting of having a relatively poor functional outcome if life can be preserved. This also indirectly contributes to the longer overall survival of both the ICA and MCA groups of patients in this study. 
When considering long-term functional outcome and mortality, factors such as age, medical comorbidities, preoperative neurological status, and laterality of the infarct may be more consequential. Patients and their families need to be counseled especially carefully when these negative prognostic factors are present. With appropriate patient selection and recent advances in neuroanaesthesia ${ }^{32,33}$ and perioperative care, ${ }^{34,35}$ the negative impression that outcomes for surgical decompression of ICA infarctions are worse than MCA infarctions can be quelled. Thus, these patients should not be given any less of a chance than a similar patient with an MCA infarction.

The main limitation of this study is its retrospective nature. In addition, there were other subgroups of patients with large territory cerebral infarctions who were not addressed in our article. These included patients who underwent prophylactic DC, who may have had a different, more favorable clinical course, and patients who received medical treatment alone, without the performance of DC. The effectiveness of DC for ICA infarction can be further assessed through direct comparison with these subgroups. There was also a small proportion of patients (ICA, 14.9\%; MCA, 14.9\%) who were lost to follow-up, mainly because of repatriation to their home countries.

This study describes the largest published modern series on ICA infarction after DC ( $\mathrm{n}=154$; ICA, 67 and MCA, 87). Other published series have had sample sizes ranging from 5 to 27 , with only 2 of the series published after $2005 .^{12,15,26-28} \mathrm{In}$ addition, despite its retrospective nature, information was readily and reliably available from electronic medical records, and patient deaths were retrieved from an external government registry that provided the exact date of all inpatient and out-of-hospital deaths. We recommend that a systematic review, or a study with a larger sample size, be performed to further validate our claim.

\section{Conclusions}

Unlike previous smaller studies, this study found that patients with malignant ICA infarction have a similar functional outcome and survival to those with MCA infarction after DC. Therefore, DC can be offered for malignant ICA infarction for life-saving purposes with limited functional recovery.

\section{References}

1. Casetta I, Fainardi E, Saia V, et al. Endovascular thrombectomy for acute ischemic stroke beyond 6 hours from onset: a real-world experience. Stroke. 2020;51(7):2051-2057.

2. Menon BK. Neuroimaging in acute stroke. Continuum (Minneap Minn). 2020;26(2):287-309.

3. Wechsler LR, Demaerschalk BM, Schwamm LH, et al. Telemedicine quality and outcomes in stroke: a scientific statement for healthcare professionals from the American heart association/American stroke association. Stroke. 2017; 48(1):e3-e25.

4. Maus V, Henkel S, Riabikin A, et al. The SAVE technique: large-scale experience for treatment of intracranial large vessel occlusions. Clin Neuroradiol. 2019;29(4):669-676.

5. Heiss WD. Malignant MCA infarction: pathophysiology and imaging for early diagnosis and management decisions. Cerebrovasc Dis. 2016;41(1-2):1-7.
6. Siegler JE, Samai A, Semmes E, Martin-Schild S. Early neurologic deterioration after stroke depends on vascular territory and stroke etiology. J Stroke. 2016;18(2):203-210.

7. Malhotra K, Goyal N, Tsivgoulis G. Internal carotid artery occlusion: pathophysiology, diagnosis, and management. Curr Atheroscler Rep. 2017;19(10):41.

8. Hong JH, Kang J, Jang MU, et al. Recanalization therapy for internal carotid artery occlusion presenting as acute ischemic stroke. J Stroke Cerebrovasc Dis. 2014;23(8):2183-2189.

9. Mpotsaris A, Kabbasch C, Borggrefe J, et al. Stenting of the cervical internal carotid artery in acute stroke management: The Karolinska experience. Interv Neuroradiol. 2017;23(2): 159-165.

10. Jüttler E, Unterberg A, Woitzik J, et al. Hemicraniectomy in older patients with extensive middle-cerebral-artery stroke. $N$ Engl J Med. 2014;370(12):1091-1100.

11. White OB, Norris JW, Hachinski VC, Lewis A. Death in early stroke, causes and mechanisms. Stroke. 1979;10(6):743.

12. Walz B, Zimmermann C, Böttger S, Haberl RL. Prognosis of patients after hemicraniectomy in malignant middle cerebral artery infarction. J Neurol. 2002;249(9):1183-1190.

13. Schwab S, Steiner T, Aschoff A, et al. Early hemicraniectomy in patients with complete middle cerebral artery infarction. Stroke. 1998;29(9):1888-1893.

14. Leonhardt G, Wilhelm H, Doerfler A, et al. Clinical outcome and neuropsychological deficits after right decompressive hemicraniectomy in MCA infarction. J Neurol. 2002;249(10): 1433-1440.

15. Holtkamp M, Buchheim K, Unterberg A, et al. Hemicraniectomy in elderly patients with space occupying media infarction: improved survival but poor functional outcome. $J$ Neurol Neurosurg Psychiatry. 2001;70(2):226-228.

16. Foerch C, Lang JM, Krause J, et al. Functional impairment, disability, and quality of life outcome after decompressive hemicraniectomy in malignant middle cerebral artery infarction. J Neurosurg. 2004;101(2):248-254.

17. Warner JJ, Harrington RA, Sacco RL, Elkind MSV. Guidelines for the early management of patients with acute ischemic stroke: 2019 update to the 2018 guidelines for the early management of acute ischemic stroke. Stroke. 2019; 50(12):3331-3332.

18. Mohan Rajwani K, Crocker M, Moynihan B. Decompressive craniectomy for the treatment of malignant middle cerebral artery infarction. Br J Neurosurg. 2017;31(4):401-409.

19. Das S, Mitchell P, Ross N, Whitfield PC. Decompressive hemicraniectomy in the treatment of malignant middle cerebral artery infarction: a meta-analysis. World Neurosurg. 2019;123:8-16.

20. Vahedi K, Vicaut E, Mateo J, et al. Sequential-design, multicenter, randomized, controlled trial of early decompressive craniectomy in malignant middle cerebral artery infarction (DECIMAL Trial). Stroke. 2007;38(9):2506-2517.

21. Jüttler E, Schwab S, Schmiedek P, et al. Decompressive surgery for the treatment of malignant infarction of the middle cerebral artery (DESTINY): a randomized, controlled trial. Stroke. 2007;38(9):2518-2525.

22. Hofmeijer J, Kappelle LJ, Algra A, et al. Surgical decompression for space-occupying cerebral infarction (the Hemicraniectomy After Middle Cerebral Artery infarction with Life-threatening Edema Trial [HAMLET]): a multicentre, open, randomised trial. Lancet Neurol. 2009;8(4):326-333.

23. Vahedi K, Hofmeijer J, Juettler E, et al. Early decompressive surgery in malignant infarction of the middle cerebral artery: a pooled analysis of three randomised controlled trials. Lancet Neurol. 2007;6(3):215-222.

24. Park JO, Park DH, Kim SD, et al. Surgical treatment for acute, severe brain infarction. J Korean Neurosurg Soc. 2007; 42(4):326-330.

25. Chen CC, Cho DY, Tsai SC. Outcome of and prognostic fac- 
tors for decompressive hemicraniectomy in malignant middle cerebral artery infarction. J Clin Neurosci. 2007;14(4): 317-321.

26. Yoo SH, Kim TH, Shin JJ, et al. The clinical efficacy of decompressive craniectomy in patients with an internal carotid artery territory infarction. J Korean Neurosurg Soc. 2012; 52(4):293-299.

27. Kilincer C, Asil T, Utku U, et al. Factors affecting the outcome of decompressive craniectomy for large hemispheric infarctions: a prospective cohort study. Acta Neurochir (Wien). 2005;147(6):587-594.

28. Daou B, Kent AP, Montano M, et al. Decompressive hemicraniectomy: predictors of functional outcome in patients with ischemic stroke. J Neurosurg. 2016;124(6):1773-1779.

29. Wang KW, Chang WN, Ho JT, et al. Factors predictive of fatality in massive middle cerebral artery territory infarction and clinical experience of decompressive hemicraniectomy. Eur J Neurol. 2006;13(7):765-771.

30. First RB. Opening the "black box": functions of the frontal lobes and their implications for sociology. Front Sociol. 2019; 4:3.

31. Yang X, Gao M, Shi J, et al. Modulating the activity of the DLPFC and OFC has distinct effects on risk and ambiguity decision-making: a tDCS study. Front Psychol. 2017;8:1417.

32. Welch Tl, Pasternak JJ. Recent advances in neuroanesthesiology. In: Prabhakar H, ed. Essentials of Neuroanesthesia. Academic Press; 2017:897-905.

33. John Doyle D, Dahaba AA, LeManach Y. Advances in anesthesia technology are improving patient care, but many challenges remain. BMC Anesthesiol. 2018;18(1):39.

34. Badenes R, Prisco L, Maruenda A, Taccone FS. Criteria for Intensive Care admission and monitoring after elective craniotomy. Curr Opin Anaesthesiol. 2017;30(5):540-545.

35. Sriganesh K, Syeda S, Shanthanna H, et al. Effect of opioid versus non-opioid analgesia on surgical pleth index and biomarkers of surgical stress during neurosurgery for brain tumors: preliminary findings. Neurol India. 2020;68(5): 1101-1105.

36. Kelly AG, Hoskins KD, Holloway RG. Early stroke mortality, patient preferences, and the withdrawal of care bias. Neurology. 2012;79(9):941-944.

37. Creutzfeldt CJ, Becker KJ, Weinstein JR, et al. Do-not-attempt-resuscitation orders and prognostic models for intraparenchymal hemorrhage. Crit Care Med.2011;39(1):158-162.

38. McCracken DJ, Lovasik BP, McCracken CE, et al. Freret al. The intracerebral hemorrhage score: a self-fulfilling prophecy? Neurosurgery. 2019;84(3):741-748.

39. Knecht S, Dräger B, Deppe M, et al. Handedness and hemispheric language dominance in healthy humans. Brain. 2000; 123(Pt 12):2512-2518.
40. Banks JL, Marotta CA. Outcomes validity and reliability of the modified Rankin scale: implications for stroke clinical trials: a literature review and synthesis. Stroke. 2007;38: 1091-1096.

41. Honeybul S, Gillett GR, Ho K. Futility in neurosurgery: a patient-centered approach. Neurosurgery. 2013;73(6):917-922.

42. Sturm JW, Donnan GA, Dewey HM, et al. Quality of life after stroke: the North East Melbourne stroke incidence study (NEMESIS). Stroke. 2004;35(10):2340-2345.

43. Rahme R, Zuccarello M, Kleindorfer D, et al. Decompressive hemicraniectomy for malignant middle cerebral artery territory infarction: is life worth living? J Neurosurg. 2012;117(4): 749-754.

44. Woertgen C, Erban P, Rothoerl RD, et al. Quality of life after decompressive craniectomy in patients suffering from supratentorial brain ischemia. Acta Neurochir (Wien). 2004;146(7): 691-695.

45. Sahadevan S, Pang WS. Do-not-resuscitate orders: towards a policy in Singapore. Singapore Med J. 1995;36(3):267-270.

46. Phua J, Kee ACL, Tan A, et al. End-of-life care in the general wards of a Singaporean hospital: an Asian perspective. J Palliat Med. 2011;14(12):1296-1301.

47. Lee L, Lo YT, See AAQ, et al. Long-term recovery profile of patients with severe disability or in vegetative states following severe primary intracerebral hemorrhage. J Crit Care. 2018;48:269-275.

\section{Disclosures}

The authors report no conflict of interest concerning the materials or methods used in this study or the findings specified in this paper.

\section{Author Contributions}

Conception and design: Lee. Acquisition of data: Lim, Vedicherla, Chan, Primalani, Tan. Analysis and interpretation of data: Lim. Drafting the article: Lim, Primalani, Lee. Critically revising the article: Lim, Lee. Reviewed submitted version of manuscript: all authors. Approved the final version of the manuscript on behalf of all authors: Lim. Statistical analysis: Lim, Saffari. Study supervision: Lee.

\section{Correspondence}

Jia Xu Lim: National Neuroscience Institute, Singapore. jiaxu. lim@mohh.com.sg. 